were used to assess the severity of symptoms (patient assessment of constipation symptoms (PAC-SYM)), somatic symptoms (patient health questionnaire (PHO-12-SS)) and the personality trait of neuroticism (big five inventory-neuroticism scale (BFI-N)). At follow up, clinical response was defined as the proportion of patients achieving 3 or more SCBM per week.

Results 64 patients (59 female, mean age 48.3 years, range 19-83) had a mean SCBM per week of 1.6 (range 0.5-2). At a mean follow up of 4.6 weeks (range 4-8) 40/64 (62.5\%) patients achieved clinical response. $8 / 64(12.5 \%)$ did not tolerate treatment due to side effects. In an intention to treat analysis, mean SCBM per week increased from 1.6 to $3.2(p=0.01)$ with mean PAC-SYM scores reducing from 27.7 to $20(p=0.001)$. Logistic regression analysis demonstrated that BFI-N (odds ratio 8.7, 95\% confidence interval (CI), 1.99-64, $p<0.01$ ) and slow transit constipation (STC) (odds ratio 1.4, 95\% CI, 1.20-2.1, $p<0.01$ ) were independently associated with positive treatment outcomes.

Conclusion Prucalopride is a useful, generally well tolerated, treatment for the management of CC in secondary care. These data suggest that efficacy could be enhanced by targeting patients with STC and in those who are more neurotic. Further work is now warranted to confirm these findings in a larger cohort of patients.

Disclosure of Interest A. Farmer Paid Instructor for: Shire Pharmaceuticals, P. Harvey: None Declared, D. Haldar: None Declared, N. Quraishi: None Declared, Q. Aziz: None Declared

\section{PTU-124 THE ASSOCIATION OF THE JOINT HYPERMOBILITY SYNDROME WITH FUNCTIONAL GASTROINTESTINAL DISORDERS - AN INTERESTING NEW FINDING THAT MAY EXPLAIN AETIOLOGY}

doi:10.1136/gutjnl-2013-304907.214

1."A Fikree, ${ }^{1} \mathrm{R}$ Aktar, ${ }^{1} \mathrm{~K}$ V Gillespie, 'L E Glasgow, ${ }^{2} \mathrm{~J}$ K Morris, ${ }^{3} \mathrm{~A}$ Farmer, ${ }^{4} \mathrm{R}$ Grahame, ${ }^{1} \mathrm{C}$ H Knowles, ${ }^{1} \mathrm{O}$ Aziz. ${ }^{1}$ Neurogastroenterology; ${ }^{2}$ Wolfson Institute, Barts and the London School of Medicine and Dentistry, Queen Mary University London, London; ${ }^{3}$ Gastroenterology, Shrewsbury and Telford NHS Trust, Shrewsbury; "Rheumatology, University College Hospital NHS Trust, London, UK

Introduction Functional Gastrointestinal Disorders (FGID) are common, but their cause is unknown. Joint hypermobility syndrome (JHS) is a common non-inflammatory connective tissue disorder characterised by joint hyperflexibility. It is associated with gastrointestinal (GI) symptoms (1), in particular unexplained symptoms (2). The association between JHS and FGID has never been studied.

Methods A nested case control study in patients aged 18-70 attending secondary care was performed. 694 consecutive new referrals to GI clinics were assessed for JHS using the Brighton criteria, prior to their outpatient consultation. Subsequent investigation by their gastroenterologist led to a diagnosis that was functional, organic or gastrooesophageal reflux (GOR); the latter were excluded due to the mixed aetiology of reflux. The control group consisted of 92 patients referred to secondary care for non-GI symptoms-those with diabetes, pregnancy, neuromuscular disorders or inflammatory arthritis were excluded. Controls were similarly assessed for JHS. JHS prevalence was compared in patients with FGID, organic GI disorders, and controls.

Results Of the 694 GI patients, 26 had GOR and 52 had not received a diagnosis-these were excluded. Thus 616 GI patients were included in the study: 363 had FGID, 253 had organic disorders. There were no significant age or gender differences between FGID and controls (age: $40.3 \pm 0.69$ vs $42.7 \pm 1.5 ; 64 \%$ vs $67 \%$ females). Compared to FGID patients, organic patients were older $(43.9 \pm 0.92$ vs $40.3 \pm 0.69$, p:0.002) and less likely to be female (54\% vs $64 \%$, p:0.008). The prevalence of JHS in FGID patients in secondary care was $40.5 \%$. This was significantly higher than in organic GI patients (26.9\%, p:0.000) and in controls (25\%, p:0.006).
Even after adjusting for age and gender differences, JHS was significantly associated with FGID (p:0.005).

Conclusion This is the first study that demonstrates a strong association between JHS and FGID, as compared to both organic GI and non-GI conditions. This suggests a potential connective tissue aetiology for $40 \%$ of FGID patients in secondary care. Furthermore, the high prevalence of JHS in FGID suggests that this common diagnosis is often overlooked. Our results have implications for future FGID research and efforts must now be focused to determine the mechanism of symptoms and identification of appropriate treatments for this subgroup of patients.

Disclosure of Interest None Declared

\section{REFERENCES}

1. Castori, M., et al., Am J Med Genet A, 2010. 152A(3): p. 556-64.

2. Zarate, N., et al., Neurogastroenterol Motil, 2010. 22(3):252-e78.

\section{PTU-125 JOINT HYPERMOBILITY IS A RISK FACTOR FOR OESOPHAGEAL HYPERSENSITIVITY}

doi:10.1136/gutjnl-2013-304907.215

1.*A Fikree, 'D Annan, 'E Woo, 'P Balendran, 'J Choy, 'J Jafari, 'E Yazaki, 'Q Aziz, 'D Sifrim. 'Neurogastroenterology, Barts and the London School of Medicine and Dentistry, Queen Mary University London, London, UK

Introduction Generalised joint hypermobility (HM) refers to an increased flexibility of the joints which is present in $5-17 \%$ of the population, and is assessed by clinical examination or validated hypermobility questionnaire. HM is associated with increased incidence of somatic pain and hypersensitivity, but its relationship with visceral hypersensitivity is unknown. Gastro-oesophageal reflux symptoms occur in over $50 \%$ of hypermobile patients (1). We hypothesised that we would observe a higher prevalence of HM in patients with hypersensitive oesophagus compared to patients with either erosive (GERD) or non erosive (NERD) reflux disease, functional heartburn, or a healthy control group.

Methods A cross sectional study of patients attending our GI physiology unit for investigation of reflux symptoms between Jan 2010 and March 2012 was undertaken. Patients completed the validated joint hypermobility questionnaire; scores $\geq 2$ out of 5 represented HM (Hakim 2003). Information from gastrosocopy and physiology testing was used to determine whether patients had GERD, NERD, hypersensitive oesophagus, or functional heartburn. Patients who were on PPI were excluded. Hypermobility questionnaire data from a group of 250 healthy volunteers was obtained from another study.

Results 457 (59\% female, age range: 15-79) patients with complete data were included. HM was present in $79(17 \%)$ patients. The prevalence of HM was highest in patients diagnosed with hypersensitive oesophagus (31\%) and lowest in NERD (15\%)-see table 1 . The prevalence of HM was significantly higher in hypersensitive oesophagus $(31 \%)$ compared to the combined prevalence in other reflux diagnoses $(16 \%), p=0.009$, and to the healthy control group $(18 \%)$, $\mathrm{p}=0.04$.

\section{Abstract PTU-125 Table 1 Prevalence of HM (\%) in different groups}

\begin{tabular}{lll}
\hline & Total number & Number with HM (score $\geq 2)(\%)$ \\
\hline GERD & 53 & $11(19 \%)$ \\
NERD & 233 & $35(15 \%)$ \\
Hypersensitive oesophagus & 49 & $15(31 \%)$ \\
Functional heartburn & 122 & $20(16 \%)$ \\
Healthy volunteers & 250 & $45(18 \%)$ \\
\hline
\end{tabular}

Conclusion $17 \%$ of patients with reflux symptoms severe enough to warrant physiology investigation have HM, which is similar to the prevalence in healthy controls. The prevalence of HM in patients 